Cite this: Mol. BioSyst., 2014, 10, 1568

Received 21st November 2013, Accepted 27th March 2014

DOI: $10.1039 / \mathrm{c} 3 \mathrm{mb} 70518 \mathrm{~h}$

www.rsc.org/molecularbiosystems

\section{A cyclic dinucleotide containing 2-aminopurine is a general fluorescent sensor for c-di-GMP and $3^{\prime}, 3^{\prime}-c G A M P \dagger$}

\author{
Benjamin T. Roembke, Jie Zhou, Yue Zheng, David Sayre, Allan Lizardo, \\ Laurentee Bernard and Herman O. Sintim*
}

\begin{abstract}
Cyclic dinucleotides have emerged as second messengers that regulate diverse processes in bacteria, as well as regulating the production of type I interferons in metazoans. Fluorescent sensors for these important second messengers are highly sought-after for high-throughput inhibitor discovery, yet most sensors reported to date are not amenable for high-throughput screening purposes. Herein, we demonstrate that a new analog, $3^{\prime}, 3^{\prime}-c G(d 2 A P) M P$, which is a 2-aminopurine (2AP)-containing cyclic dinucleotide, self-associates in the presence of $\mathrm{Mn}^{2+}$ with an association constant of $120000 \mathrm{M}^{-1} \cdot 3^{\prime} 3^{\prime}-\mathrm{cG}(\mathrm{d} 2 \mathrm{AP}) \mathrm{MP}$ can also form a heterodimer with CGAMP, activator of immune regulator, STING, or the bacterial biofilm regulator, c-di-GMP in the presence of $\mathrm{Mn}(I)$. Upon dimer formation, the fluorescence of $3^{\prime}, 3^{\prime}-\mathrm{cG}(\mathrm{d} 2 \mathrm{AP}) \mathrm{MP}$ is quenched and this provides a convenient method to monitor the enzymatic processing of both DGC and PDE enzymes, opening up several opportunities for the discovery of inhibitors of nucleotide signaling.
\end{abstract}

\section{Introduction}

Cyclic dinucleotides (Fig. 1) have piqued the interests of scientists of different disciplines in the last few years due to the increasingly diverse processes that they regulate in both prokaryotes and eukaryotes. In bacteria, c-di-GMP and c-di-AMP have been shown to regulate multitudes of processes, including antibiotic resistance, ${ }^{1}$ cell wall remodeling, ${ }^{2}$ biofilm formation, ${ }^{3}$ virulence or toxin production, ${ }^{4}$ resistance to heavy metals and others. ${ }^{5}$ These bacterial second messengers achieve their biological effect via binding to proteins ${ }^{5}$ and RNA riboswitches. ${ }^{6-9}$ Recently it was shown that bacteria also produce the mixed cyclic dinucleotide, $3^{\prime}, 3^{\prime}$-cGAMP $\left(3^{\prime}, 3^{\prime}\right.$ implies that both $\mathrm{G}$ and $\mathrm{A}$ have a $3^{\prime} 5^{\prime}$-phosphodiester linkage) and that $3^{\prime}, 3^{\prime}$-cGAMP affects bacterial chemotaxis and colonization. ${ }^{10}$ Eukaryotes have not been shown yet to produce c-di-GMP or c-di-AMP but these molecules affect eukaryotic physiology nonetheless via binding to the immune modulator STING. ${ }^{11,12}$ Recently it has been shown that eukaryotes also produce $2^{\prime}, 3^{\prime}$-cGAMP (containing $2^{\prime}, 5^{\prime}$ and $3^{\prime}, 5^{\prime}$ linkages). ${ }^{13,14}$ cGAMP production by cGAMP synthase is initiated when DNA is present in the cytosol (which usually implies the presence of a foreign genetic material or even self DNA, both of which signifies danger). The produced cGAMP then binds to

Department of Chemistry and Biochemistry, University of Maryland, College Park, MD 20742,USA. E-mail: hsintim@umd.edu

$\dagger$ Electronic supplementary information (ESI) available. See DOI: 10.1039/ c3mb70518h
STING to activate the production of type 1 interferons. ${ }^{15}$ The newly discovered roles for cyclic dinucleotides in both prokaryotes and eukaryotes have provided new targets to develop therapeutics against diseased states ranging from cancer, autoimmune disease, bacterial infection and even viral infection, such as HIV. ${ }^{16}$ Consequently new tools that would facilitate the specific detection of these cyclic dinucleotides would aid studies that aim to understand how environmental conditions (including nutrients, antibiotics) affect the intracellular production or degradation of these nucleotides and also could be used to discover new small molecules that inhibit cyclic dinucleotide metabolism proteins.

Few methods have been developed for the detection of cyclic dinucleotides or to monitor the synthesis or degradation of cyclic dinucleotides. ${ }^{1,19-29}$ Furthermore, these assays utilize radioactive TLC (for all dinucleotides for which the ${ }^{32} \mathrm{P}$-labeled form can be synthesized via enzymatic means), HPLC-MS/MS (c-di-GMP/c-di-AMP $/ 3^{\prime}, 3^{\prime}$-cGAMP), ${ }^{19}$ circular dichroism (c-diGMP), ${ }^{20}$ conditional riboswitch (c-di-GMP), ${ }^{21,22,28}$ or intercalatorbased methods that utilize the ability of c-di-GMP to form G-quadruplexes. ${ }^{23-26}$ These aforementioned methods, although sensitive, either use expensive instrumentation that might not be readily accessible for biologists researching nucleotide signaling or are difficult/inconvenient to use. The thiazole orange assay, which was disclosed by us, is simple to use but requires high concentrations of monovalent cations and a long incubation time to form the fluorescent c-di-GMP aggregate so it is most appropriate for end-point, but not real-time, assay. A fluorescent riboswitch assay, using Spinach riboswitch, to 


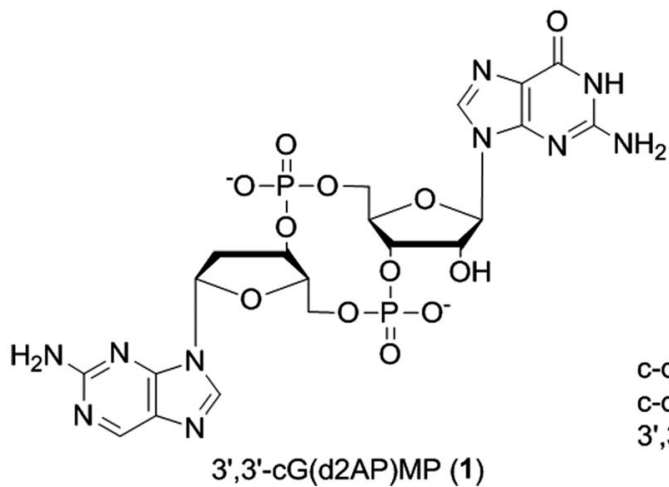

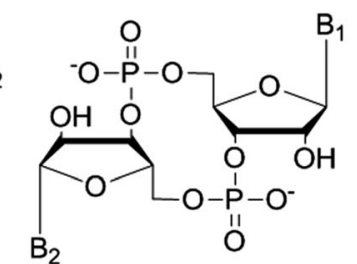

$\begin{array}{lll}\text { c-di-GMP } & \text { (2) } \mathrm{B}_{1}=\mathrm{B}_{2}=\text { Guanine } \\ \text { c-di-AMP } & \text { (3) } \mathrm{B}_{1}=\mathrm{B}_{2}=\text { Adenine } \\ \text { 3',3'-cGAMP (4) } \mathrm{B}_{1}=\text { Guanine }\end{array}$

$\mathrm{B}_{2}=$ Adenine

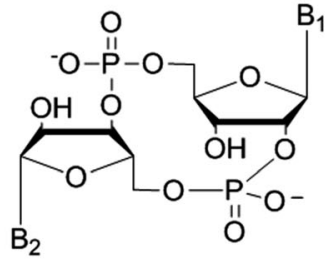

2',3'-cGAMP (5) $B_{1}=$ Guanine

$\mathrm{B}_{2}=$ Adenine

2',3'-cAGMP (6) $B_{1}=$ Adenine

$\mathrm{B}_{2}=$ Guanine

Fig. 1 Cyclic dinucleotides, used in this study, were synthesized following literature precedents. ${ }^{13,17,18}$ See ESI $\dagger$ for the synthesis and characterization of $3^{\prime}, 3^{\prime}-c G(d 2 A P) M P$.

detect c-di-GMP was also disclosed by $\mathrm{us}^{21}$ and this method has been adapted for the in vivo detection of c-di-GMP by others. ${ }^{28}$ Although this assay is sensitive, it utilizes an RNA sensor, which has to be handled carefully to avoid adventitious degradation by RNAses. An interesting whole-cell assay to detect c-di-GMP has also been described. ${ }^{29} \mathrm{~A}$ complementary assay that does not utilize proteins or transformation (since not all bacterial strains can be readily transformed with plasmids) and which can be used in real time to monitor enzymatic activity could be broadly useful for the cyclic dinucleotide signaling field.

\section{Results and discussion}

We herein describe a convenient assay for the detection of cyclic dinucleotides that can be used in real time to monitor the enzymatic activities of cyclic dinucleotide synthases or phosphodiesterases and hence has the potential to be used in highthroughput screening assays for inhibitors of these enzymes. Cyclic dinucleotides are known to form self-intercalated dimers and divalent cations are known to promote the dimerization of cyclic dinucleotides. ${ }^{30}$ We therefore hypothesized that the two nucleobases of a 2-aminopurine (2AP) analog of $3^{\prime}, 3^{\prime}$-cGAMP $\left(3^{\prime}, 3^{\prime}-\mathrm{cG}(\mathrm{d} 2 \mathrm{AP}) \mathrm{MP}, 1\right)$ cannot stack with each other because the distance between the nucleobases of a $3^{\prime}, 3^{\prime}$-cyclic dinucleotide is $\sim 6.7 \AA,^{31}$ which is too far for effective orbital overlap for $\pi-\pi$ stacking interaction. However upon dimerization with self or other cyclic dinucleotides, the 2AP nucleobase in $3^{\prime}, 3^{\prime}-\mathrm{cG}(\mathrm{d} 2 \mathrm{AP}) \mathrm{MP}$ would $\pi$-stack with another nucleobase and the fluorescence of the 2AP would be quenched (see Fig. 2). ${ }^{32-34}$ Therefore at low concentrations of $3^{\prime}, 3^{\prime}-\mathrm{cG}(\mathrm{d} 2 \mathrm{AP}) \mathrm{MP}$, when it will mainly exist in the monomeric form, it would form heterodimers with other natural cyclic dinucleotides and the heterodimerization could be detected via fluorescence reduction.

The fluorescence of $10 \mu \mathrm{M} \mathrm{3} 3^{\prime}, 3^{\prime}-\mathrm{cG}(\mathrm{d} 2 \mathrm{AP}) \mathrm{MP}$ in the presence of $\mathrm{Mn}^{2+}(15 \mathrm{mM})$ was $50 \%$ less than that in the presence of monovalent cations $\left(\mathrm{Li}^{+}, \mathrm{K}^{+}, \mathrm{NH}_{4}{ }^{+}, \mathrm{Cs}^{+}\right)$or $\mathrm{Mg}^{2+}$ or when no cation was present (Fig. 3A), suggesting that $\mathrm{Mn}^{2+}$ promoted the dimerization of $3^{\prime}, 3^{\prime}-\mathrm{cG}(\mathrm{d} 2 \mathrm{AP}) \mathrm{MP}$. This was in line with earlier

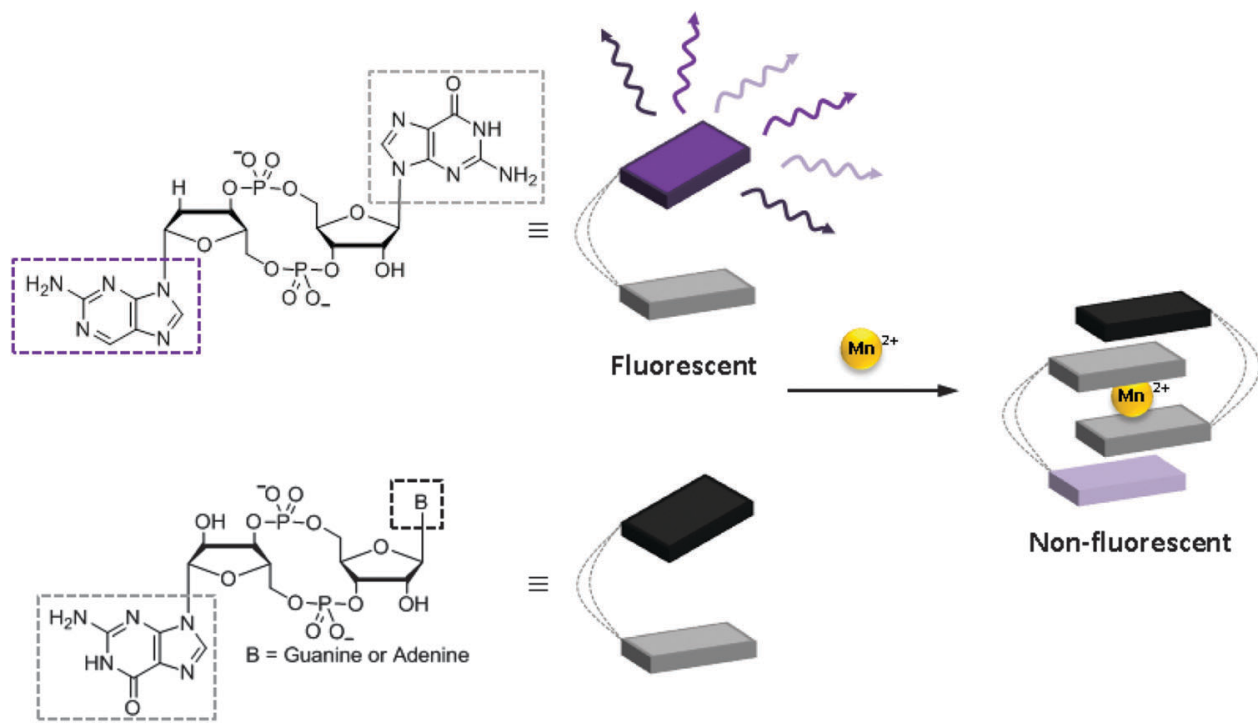

Fig. 2 Our working hypothesis is that the 2AP cyclic dinucleotide would form heterodimers with natural cyclic dinucleotide and this will result in fluorescence quenching. 

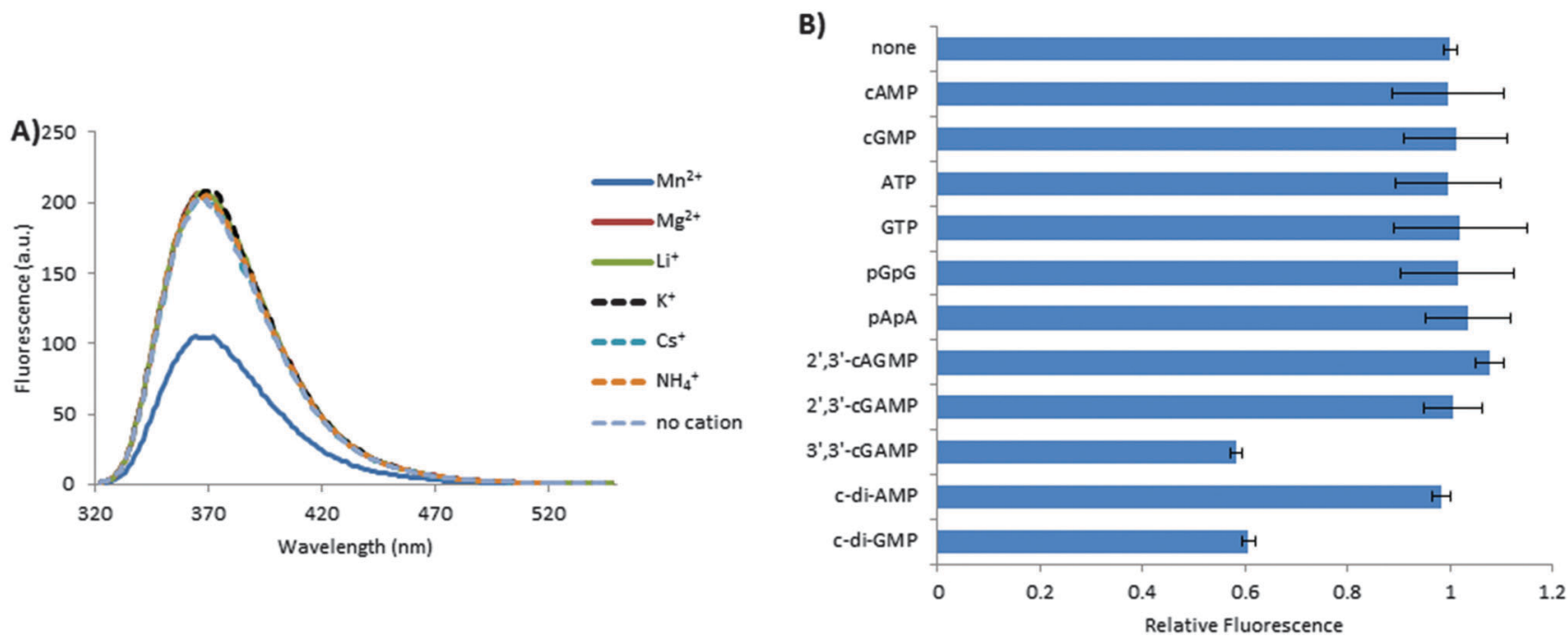

Fig. 3 (A) The fluorescence of $c G(d 2 A P) M P$ in the presence of different cations; (B) relative fluorescence intensity of $c G(d 2 A P) M P$ in the presence of different linear and cyclic nucleotides. Conditions: (A) $10 \mu \mathrm{M} \mathrm{cG(d2AP)MP,} 15 \mathrm{mM}$ divalent salt or $250 \mathrm{mM}$ monovalent salt in $50 \mathrm{mM}$ Tris- $\mathrm{HCl}$ buffer ( $\mathrm{pH}=7.5$ ). (B) $1 \mu \mathrm{M} \mathrm{cG(d2AP)MP,} 10 \mu \mathrm{M}$ cyclic or linear nucleotide, $15 \mathrm{mM} \mathrm{MnCl}_{2}$ in $50 \mathrm{mM}$ Tris- $\mathrm{HCl}$ buffer $(\mathrm{pH}=7.5)$. Excitation $=310 \mathrm{~nm}$. Fluorescence emission was recorded at $369 \mathrm{~nm}$ and compared to the emission of cG(d2AP)MP without any added nucleotide to give relative fluorescence.

observation by Cutruzzola and co-workers who showed that c-di-GMP also forms self-intercalated dimer, which could be observed in a CD spectrum, in the presence of $\mathrm{Mn}^{2+} .{ }^{20}$ Without direct evidence, we cannot conclude with certainty that the inability of $\mathrm{Mg}^{2+}$ or monovalent cations to cause fluorescent quenching in $3^{\prime}, 3^{\prime}-\mathrm{cG}(\mathrm{d} 2 \mathrm{AP}) \mathrm{MP}$ is because they do not promote dimerization. It is however known that the fluorescence of 2-aminopurine is quenched upon stacking $^{35}$ so it is probably that at the tested concentration $(10 \mu \mathrm{M})$, neither $\mathrm{Mg}^{2+}$ nor the tested monovalent cations facilitated significant dimerization of $3^{\prime}, 3^{\prime}-\mathrm{cG}(\mathrm{d} 2 \mathrm{AP}) \mathrm{MP}$. Secondly, Gentner et al. have demonstrated, using NMR, that the dissociation constant of a similar dinucleotide, c-di-GMP, in the presence of $\mathrm{Mg}^{2+}$ is $520 \pm$ $10 \mu \mathrm{M}$, which implies that cyclic dinucleotides that associate with similar association constants would mainly exist as monomers at $10 \mu \mathrm{M}$, in the presence of $\mathrm{Mg}^{2+36}$. Next, we investigated the fluorescence of $1 \mu \mathrm{M} 3^{\prime}, 3^{\prime}-\mathrm{cG}(\mathrm{d} 2 \mathrm{AP}) \mathrm{MP}$ in the presence of $10 \mu \mathrm{M}$ of several biologically relevant nucleotides (both linear and cyclic mono- and dinucleotides; see Fig. 3B). Interestingly both c-di-GMP and $3^{\prime}, 3^{\prime}$-cGAMP, but not $2^{\prime}, 3^{\prime}$-cGAMP or $2^{\prime}, 3^{\prime}$-cAGMP or c-di-AMP, decreased the fluorescence of $3^{\prime}, 3^{\prime}-\mathrm{cG}(\mathrm{d} 2 \mathrm{AP}) \mathrm{MP}$ (see Fig. 3B). Linear dinucleotides, pGpG and pApA, as well as all mononucleotides tested (cGMP, cAMP, ATP and GTP) did not reduce the fluorescence of the 2AP analog of cGAMP. It appears that at least one guanine nucleobase is required for the formation of the heterodimer with $3^{\prime}, 3^{\prime}-\mathrm{cG}(\mathrm{d} 2 \mathrm{AP}) \mathrm{MP}$. In addition to the requirement for at least one guanine, circularization of the dinucleotide is also a requirement for dimerization because the linear $\mathrm{pGpG}$, which contains the same number of guanines as c-di-GMP, did not affect the fluorescence of $3^{\prime}, 3^{\prime}-\mathrm{cG}(\mathrm{d} 2 \mathrm{AP}) \mathrm{MP}$. We have previously noted that circularization remarkably affects the propensity of dinucleotides to form polymorphs. For example, whereas c-di-GMP could form G-quadruplexes in the presence of aromatic intercalators at low micromolar concentrations, the linear form (pGpG) did not form G-quadruplexes at similar conditions or even when the concentration of the linear form was raised by orders of magnitude. ${ }^{23,24,37} \mathrm{CD}$ spectroscopy has been proposed as a good tool to investigate the dimerization of cyclic dinucleotides. Cutruzzola and co-workers demonstrated that increase in CD intensity, especially between the $260-280 \mathrm{~nm}$ region is an indication of dimerization. ${ }^{20}$ We therefore used $\mathrm{CD}$ to investigate if the reduction of the fluorescence of $3^{\prime}, 3^{\prime}-\mathrm{cG}(\mathrm{d} 2 \mathrm{AP}) \mathrm{MP}$ by cyclic dinucleotides correlated with propensity to form selfdimers as judged by hyperchromicity in the CD bands between 260 and $280 \mathrm{~nm}$. In the presence of $\mathrm{Mn}^{2+}, 3^{\prime}, 3^{\prime}$-cGAMP, c-di-GMP and the fluorescent analog $3^{\prime}, 3^{\prime}-\mathrm{cG}(\mathrm{d} 2 \mathrm{AP}) \mathrm{MP}$ formed selfintercalated dimers (compare $\mathrm{CD}$ bands in the presence and absence of $\mathrm{Mn}^{2+}$, see Fig. 4). On the other hand, at $40 \mu \mathrm{M}$ nucleotide concentration, the $\mathrm{CD}$ spectra of c-di-AMP, $2^{\prime}, 3^{\prime}$-cGAMP or $2^{\prime}, 3^{\prime}$-cAGMP did not show major differences between presence and absence of $\mathrm{Mn}^{2+}$ (see ESI, $\dagger$ Fig. S2), implying that these dinucleotides do not form significant amounts of dimers at the tested concentration $(40 \mu \mathrm{M})$. Although, c-di-AMP has been shown to form dimers at millimolar concentrations ${ }^{38}$ our data confirms earlier observation ${ }^{20}$ that in the presence of $\mathrm{Mn}^{2+}$ cyclic dinucleotides that contain at least one guanine seem to have a higher propensity to form intercalated dimers than those lacking guanine but contain adenine. Analysis of the crystal structure of dimeric c-di-GMP, in complex with $\mathrm{Mn}^{2+}$, explains why at least one guanine is needed for the effective dimerization in the presence of divalent cations. In dimeric c-di-GMP, $\mathrm{Mn}^{2+}$ coordinates with the 06 of guanine via water molecules (see ESI, $\dagger$ Fig. S3 and ref. 20). Proteins that bind to self-intercalated dimers of c-di-GMP are known $^{39}$ but c-di-AMP has not been shown to be bound to any protein in the dimer form. This could be due to the fact that because c-di-AMP is a recently discovered dinucleotide, ${ }^{40}$ in contrast to c-di-GMP that was discovered more than two decades ago. ${ }^{41}$ Many receptors for c-di-AMP have not been 

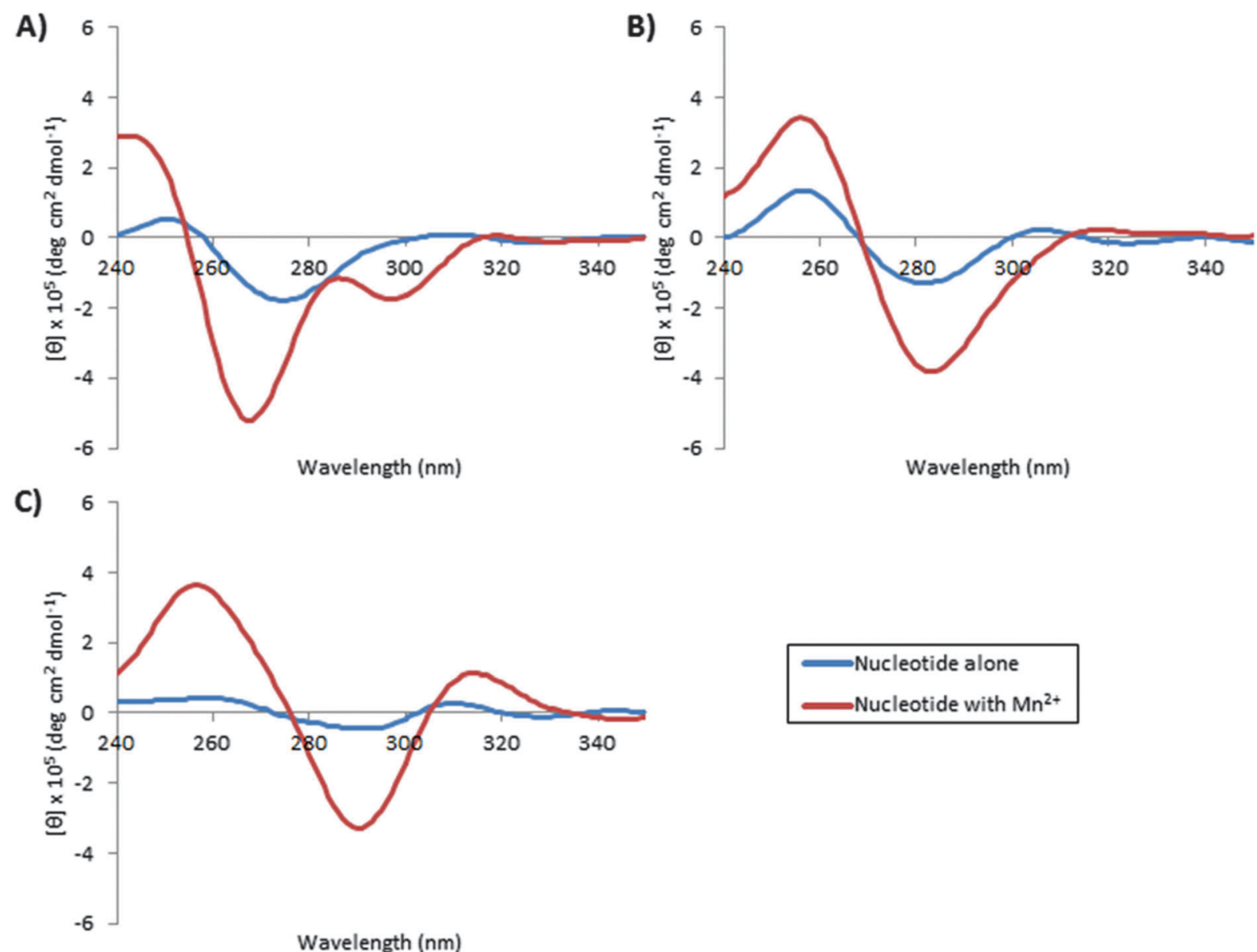

Wavelength $(\mathrm{nm})$

Fig. $4 \mathrm{CD}$ spectra of cyclic dinucleotides in the presence and absence of $\mathrm{Mn}^{2+}$. (A) $3^{\prime}, 3^{\prime}$-cGAMP; (B) C-di-GMP; (C) 3', 3'-cG(dAP)MP. Conditions: $40 \mu M$ cyclic nucleotide, $15 \mathrm{mM} \mathrm{Mn}^{2+}$ (where applicable) and $50 \mathrm{mM}$ Tris- $\mathrm{HCl}(\mathrm{pH}=7.5)$.

structurally characterized and that it could be just a matter of time before a receptor that binds to dimeric c-di-AMP is found.

Having established that $3^{\prime}, 3^{\prime}-\mathrm{cG}(\mathrm{d} 2 \mathrm{AP}) \mathrm{MP}$ can report the presence of c-di-GMP and $3^{\prime}, 3^{\prime}$-cGAMP, we proceeded to determine the association/dissociation constants of this cyclic dinucleotide (see Fig. 5). UV was found not to be a very sensitive method for the determination of dimerization constants of the cyclic dinucleotides studied, so we opted to use the

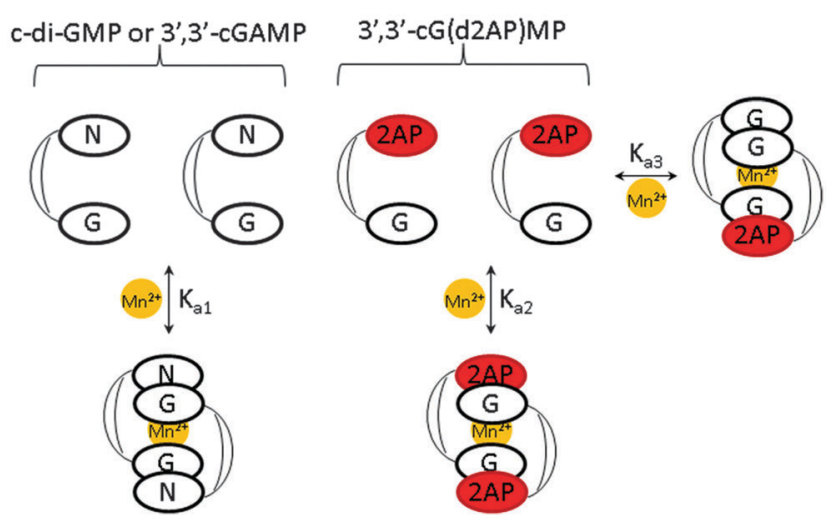

Fig. 5 Cyclic dinucleotide equilibria. fluorescence of $3^{\prime} 3-\mathrm{cG}(\mathrm{d} 2 \mathrm{AP}) \mathrm{MP}$ to determine both the homodimerization constant of $3^{\prime} 3^{\prime}-\mathrm{cG}(\mathrm{d} 2 \mathrm{AP}) \mathrm{MP}$ and the $\mathrm{IC}_{50}$ for the heterodimerization of c-di-GMP and $3^{\prime} 3^{\prime}$-cGAMP with $3^{\prime} 3^{\prime}$-cG(d2AP)MP, vide infra.

The association constant of $3^{\prime}, 3^{\prime}-\mathrm{cG}(\mathrm{d} 2 \mathrm{AP}) \mathrm{MP}$ can be defined as follows:

$$
K_{\mathrm{a} 2}=\frac{\left[2 \mathrm{AP}_{2}\right]}{[2 \mathrm{AP}]^{2}}
$$

where $[2 \mathrm{AP}]$ is the equilibrium concentration of $3^{\prime}, 3^{\prime}-\mathrm{cG}(\mathrm{d} 2 \mathrm{AP}) \mathrm{MP}$ and $\left[2 \mathrm{AP}_{2}\right]$ is the equilibrium concentration of $3^{\prime}, 3^{\prime}-\mathrm{cG}(\mathrm{d} 2 \mathrm{AP}) \mathrm{MP}$ dimer. The $\left[\mathrm{Mn}^{2+}\right]$ can be left out of the expression because $\left[\mathrm{Mn}^{2+}\right] \gg[2 \mathrm{AP}]_{\mathrm{T}}$ (the total concentration of $\left.3^{\prime}, 3^{\prime}-\mathrm{cG}(\mathrm{d} 2 \mathrm{AP}) \mathrm{MP}\right)$. If $\left[\mathrm{Mn}^{2+}\right]$ is included however, $K_{\mathrm{a} 2}$ can be expressed as such:

$$
\begin{gathered}
K_{\mathrm{a} 2}{ }^{*}=\frac{\left[2 \mathrm{AP}_{2}\right]}{[2 \mathrm{AP}]^{2}\left[\mathrm{Mn}^{2+}\right]^{n}} \\
K_{\mathrm{a} 2}=K_{\mathrm{a} 2}{ }^{*}\left[\mathrm{Mn}^{2+}\right]^{n} \\
\ln K_{\mathrm{a} 2}=\ln K_{\mathrm{a} 2}{ }^{*}+n \ln \left[\mathrm{Mn}^{2+}\right]
\end{gathered}
$$

Therefore, a plot of $\ln K_{\mathrm{a} 2}$ versus $\ln \left[\mathrm{Mn}^{2+}\right]$ will give the number of $\mathrm{Mn}^{2+}$ associated with the dinucleotide dimer. 
The total fluorescence of the system $\left(F_{\mathrm{T}}\right)$ can be decomposed into two parts, see eqn (2).

$$
F_{\mathrm{T}}=f_{1}[2 \mathrm{AP}]+f_{2}\left[2 \mathrm{AP}_{2}\right]
$$

where $f_{1}$ and $f_{2}$ are fluorescence constants of $3^{\prime}, 3^{\prime}$-cG(d2AP)MP and $3^{\prime}, 3^{\prime}-\mathrm{cG}(\mathrm{d} 2 \mathrm{AP}) \mathrm{MP}$ dimer respectively. Making appropriate substitutions (see $\mathrm{ESI} \dagger$ ),

$$
\begin{aligned}
F_{\mathrm{T}}= & \left(\frac{-1+\sqrt{1+8 K_{\mathrm{a} 2}[2 \mathrm{AP}]_{\mathrm{T}}}}{4 K_{\mathrm{a} 2}[2 \mathrm{AP}]_{\mathrm{T}}}\right)\left([2 \mathrm{AP}]_{\mathrm{T}} f_{1}-\frac{[2 \mathrm{AP}]_{\mathrm{T}} f_{2}}{2}\right) \\
& +\frac{[2 \mathrm{AP}]_{\mathrm{T}} f_{2}}{2}
\end{aligned}
$$

where $[2 \mathrm{AP}]_{\mathrm{T}}$ is the total concentration of $3^{\prime}, 3^{\prime}-\mathrm{cG}(\mathrm{d} 2 \mathrm{AP}) \mathrm{MP}$. Using eqn (3) and curve fitting software (OriginPro 8), $f_{1}, f_{2}$ and $K_{\mathrm{a} 2}$, the homodimerization constant for $3^{\prime}, 3^{\prime}-\mathrm{cG}(\mathrm{d} 2 \mathrm{AP}) \mathrm{MP}$, could be determined as $2.1 \times 10^{7}, \sim 0$ and $1.2 \pm 0.3 \times$ $10^{5} \mathrm{M}^{-1}$ respectively (see Fig. 6 and Table 2 for $K_{\mathrm{a}}$ and $K_{\mathrm{d}}$ values).

A plot of $\ln K_{\mathrm{a} 2}$ versus $\left[\mathrm{Mn}^{2+}\right]$, see Fig. 7, gave a linear graph with slope $0.59 \pm 0.01$ (i.e. the stoichiometry of bound $\mathrm{Mn}^{2+}$ ). The value $n=0.6$ is of similar order to that obtained for c-di-GMP using X-ray crystallography $(n=1) .^{20}$

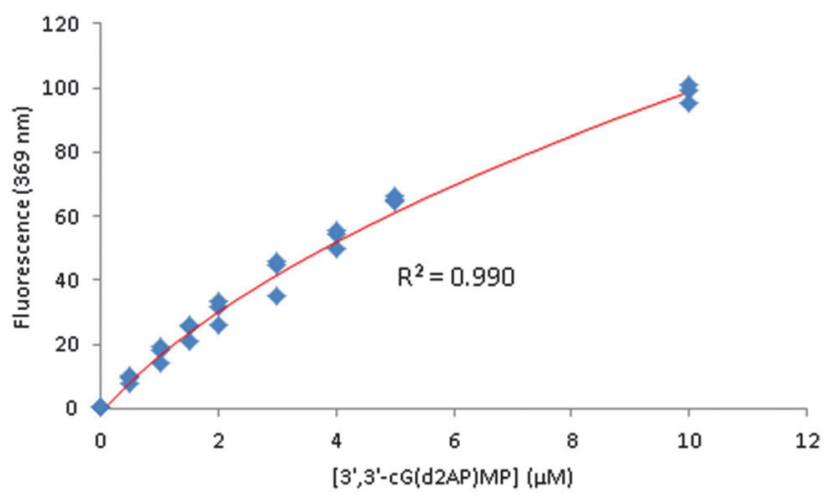

Fig. 6 A plot of fluorescence intensity versus concentration of $3^{\prime}, 3^{\prime}$ $c G(d 2 A P) M P$ in the presence of $15 \mathrm{mM} \mathrm{MnCl}_{2}$ and fitted using eqn (3) to obtain the homodimerization constant. Conditions: varying concentrations of $3^{\prime}, 3^{\prime}-\mathrm{cG}(\mathrm{d} 2 \mathrm{AP}) \mathrm{MP}, 15 \mathrm{mM} \mathrm{MnCl} 2$ in $50 \mathrm{mM}$ Tris- $\mathrm{HCl}(\mathrm{pH}=7.5)$.

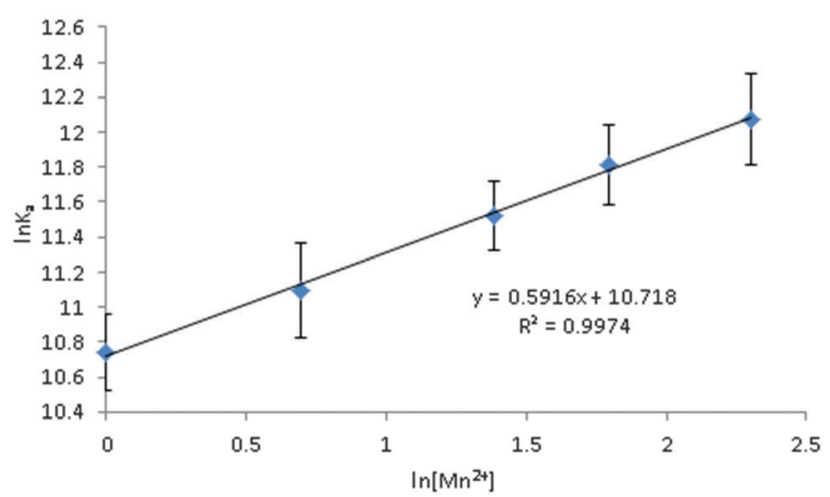

Fig. 7 A plot giving the binding stoichiometry of $\mathrm{Mn}^{2+}$ as the slope. Conditions: each binding constant was determined using varying concentrations of $\mathrm{cG}(\mathrm{d} 2 \mathrm{AP}) \mathrm{MP}, 1,2,4,6$ or $10 \mathrm{mM} \mathrm{MnCl} 2$ in $50 \mathrm{mM}$ Tris- $\mathrm{HCl}(\mathrm{pH}=7.5)$.

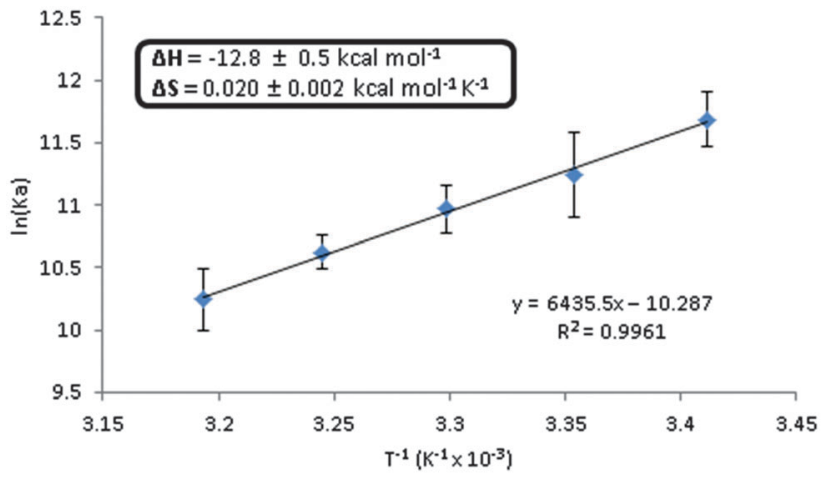

Fig. 8 Van't Hoff plot of the association constants for $3^{\prime}, 3^{\prime}-c G(d 2 A P) M P$ dimerization at different temperatures. Conditions: varying concentrations of $3^{\prime}, 3^{\prime}-\mathrm{cG}(\mathrm{d} 2 \mathrm{AP}) \mathrm{MP}, 15 \mathrm{mM} \mathrm{MnCl}$ in $50 \mathrm{mM}$ Tris- $\mathrm{HCl}(\mathrm{pH}=7.5)$. Each equilibrium constant was measured in triplicate. Samples were allowed to incubate in the cuvette at the given temperature for at $15 \mathrm{~min}$ prior to measurement.

Table 1 Association and dissociation constants for the dimerization of 3', 3'-cG(d2AP)MP

\begin{tabular}{lccl}
\hline$T(\mathrm{~K})$ & $K_{\mathrm{a}}\left(\mathrm{M}^{-1} \times 10^{5}\right)$ & $K_{\mathrm{d}}(\mu \mathrm{M})$ & $\Delta G\left(\mathrm{kcal} \mathrm{mol}^{-1}\right)$ \\
\hline 293.15 & $1.2 \pm 0.3$ & $8 \pm 2$ & -6.8 \\
298.15 & $0.8 \pm 0.3$ & $13 \pm 4$ & -6.7 \\
303.15 & $0.6 \pm 0.1$ & $17 \pm 3$ & -6.6 \\
308.15 & $0.41 \pm 0.06$ & $24 \pm 3$ & -6.5 \\
313.15 & $0.28 \pm 0.07$ & $36 \pm 9$ & -6.4
\end{tabular}

A Van't Hoff plot revealed a binding enthalpy of $-12.8 \pm$ $0.5 \mathrm{kcal} \mathrm{mol}^{-1}$ and entropy of $0.020 \pm 0.002 \mathrm{kcal} \mathrm{mol}^{-1} \mathrm{~T}^{-1}$ for $3^{\prime} 3^{\prime}$-cG(2AP)MP dimerization (see Fig. 8 and Table 1). The reaction is exergonic and enthalpy driven.

When another cyclic dinucleotide, apart from $3^{\prime} 3^{\prime}-\mathrm{cG}(\mathrm{d} 2 \mathrm{AP}) \mathrm{MP}$, is present, the total fluorescence intensity from the $2 \mathrm{AP}$ unit becomes,

$$
F_{\mathrm{T}}=f_{1}[2 \mathrm{AP}]+f_{2}\left[2 \mathrm{AP}_{2}\right]+f_{3}[2 \mathrm{AP} \cdot \mathrm{cNMP}]
$$

where $f_{3}$ is the fluorescence constant of the heterodimer between c-di-GMP or $3^{\prime}, 3^{\prime}$-cGAMP and $3^{\prime}, 3^{\prime}$-cG(d2AP)MP. $f_{2}$ has already been determined to be $\sim 0$ (from curve fitting in Fig. 6). Both $f_{2}$ and $f_{3}$ are low (the dimer forms are "darker" than the monomer form due to fluorescence quenching from $\pi-\pi$ stacking of the 2AP fluorophore). The dimer and monomer forms of c-di-GMP or $3^{\prime} 3^{\prime}$-cGAMP are also "dark" so it is nontrivial to tease out the separate association constants in Fig. 5 ( $K_{\mathrm{a} 1}$ and $K_{\mathrm{a} 3}$ ), using fluorescence data alone. Attempts to use UV titration to independently determine $K_{\mathrm{a} 1}$ failed due to the insensitivity of UV. Because $\mathrm{Mn}^{2+}$ is paramagnetic, we could not apply NMR methods to determine $K_{\mathrm{a} 1}$, as was previously demonstrated for c-di-GMP dimer formation in the presence of $\mathrm{Mg}^{2+} \cdot{ }^{36}$ We therefore proceeded to determine the $\mathrm{IC}_{50}$ of c-diGMP or $3^{\prime} 3^{\prime}$-cGAMP binding to $1 \mu \mathrm{M} 3^{\prime} 3^{\prime}$-cG(d2AP)MP. We calculated that at $1 \mu \mathrm{M}$, over $90 \%$ of $3^{\prime} 3^{\prime}-\mathrm{cG}(\mathrm{d} 2 \mathrm{AP}) \mathrm{MP}$ would exist as monomer so the $\mathrm{IC}_{50}$ values (see Table 2 ) could give a reasonable guide of the concentration ranges that these nonfluorescent analogs form dimers in the presence of $\mathrm{Mn}^{2+}$. 
Table 2 Thermodynamic parameters at $20{ }^{\circ} \mathrm{C}$ of cyclic dinucleotides used in this study

\begin{tabular}{lllll}
\hline & \multicolumn{2}{l}{ Homodimerization } & & Heterodimerization $^{a}$ \\
\cline { 2 - 3 } Nucleotide & $K_{\mathrm{d}}(\mu \mathrm{M})$ & $K_{\mathrm{a}}\left(\mathrm{M}^{-1}\right)$ & & $\mathrm{IC}_{50}(\mu \mathrm{M})$ \\
\hline $3^{\prime}, 3^{\prime}$-cG(d2AP)MP & $8 \pm 2$ & $1.2 \pm 0.3 \times 10^{5}$ & & N/A \\
c-di-GMP & N.D. & N.D. & $11 \pm 1$ \\
$3^{\prime}, 3^{\prime}$-cGAMP & N.D. & N.D. & $9 \pm 1$
\end{tabular}

${ }^{a}$ Concentration of $3^{\prime} 3^{\prime}-\mathrm{cG}(\mathrm{d} 2 \mathrm{AP}) \mathrm{MP}=1 \mu \mathrm{M}$ and at this concentration over $91 \%$ of this fluorescent probe exists as monomer. The $\mathrm{IC}_{50}$ value is a composite value of homodimerization and heterodimerization constants.

The c-di-GMP $/ 3^{\prime}, 3^{\prime}$-cGAMP concentration dependence of the fluorescence of $3^{\prime}, 3^{\prime}-\mathrm{cG}(\mathrm{d} 2 \mathrm{AP}) \mathrm{MP}$ (Fig. 9) suggested to us that $3^{\prime}, 3^{\prime}-\mathrm{cG}(\mathrm{d} 2 \mathrm{AP}) \mathrm{MP}$ could be used to monitor the synthesis of c-di-GMP by WspR (Fig. 10). For the detection of enzymatically synthesized c-di-GMP, we first performed the reaction for $30 \mathrm{~min}$ and then added the fluorescent probe and $\mathrm{Mn}^{2+}$ to detect the synthesized cyclic dinucleotide. The $\mathrm{Mn}^{2+}$ and the fluorescent probe were added after the c-di-GMP was made because WspR requires $\mathrm{Mg}^{2+}$ and not $\mathrm{Mn}^{2+}$ for the synthesis of c-di-GMP. In the presence of enzymatically synthesized c-di-GMP, the fluorescence of the 2AP probe was lower than when c-di-GMP was not made (i.e. GTP or WspR enzyme were not added to the reaction, see Fig. 10). This new assay for the detection of enzymatically produced c-di-GMP is complementary to the thiazole orange assay, previously reported by us and it does not require long incubation time, in contrast to the thiazole orange assay. ${ }^{23}$ We also hypothesized that upon cleavage of $3^{\prime}, 3^{\prime}$ cG(d2AP)MP by phosphodiesterases, the microenvironment of the 2AP moiety would change and would result in a change in fluorescence, hence this probe could be used to monitor the activity of PDE's. Snake venom phosphodiesterase, SVPD, is a general phosphodiesterase that can cleave both linear and cyclic nucleotides. SVPD can be inhibited by EDTA and so is a good model system to test if $3^{\prime}, 3^{\prime}-\mathrm{cG}(\mathrm{d} 2 \mathrm{AP}) \mathrm{MP}$ could be used to discover inhibitors of PDE. ${ }^{43}$ SVPD could cleave the probe into the linear dinucleotide and this could be monitored in real time, see Fig. 11 and ESI, $\dagger$ Fig. S5 for the HPLC analysis of the cleavage. For c-di-AMP phosphodiesterase, YybT, cleavage also occurred but this time the reaction was slower than the SVPD

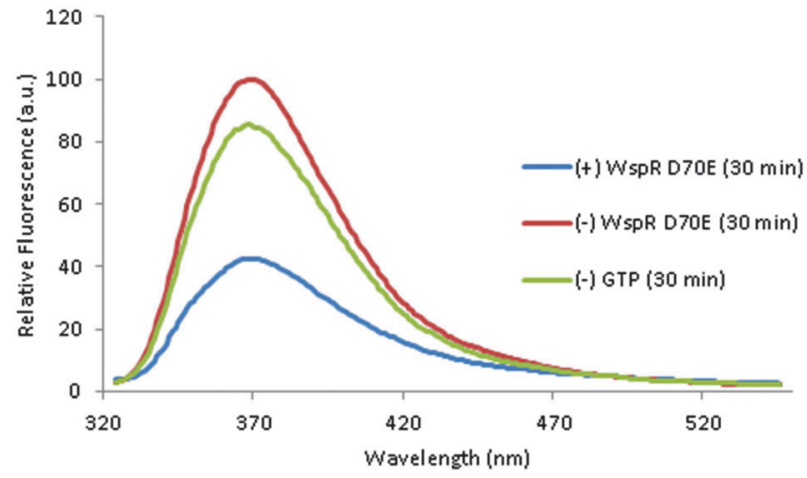

Fig. 10 The $3^{\prime} 3^{\prime}-c G(d 2 A P) M P$ probe was added to the $D G C$ reaction after 30 min, when c-di-GMP had been produced. (+) WspR D70E: contains enzyme and GTP; (-) WspR D70E contains no WsprD70E; (-) GTP contains no GTP. Enzymatic conditions: $10 \mu \mathrm{M}$ WspR D70E, $100 \mu \mathrm{M}$ GTP, $100 \mathrm{mM}$ $\mathrm{NaCl}, 5 \mathrm{mM} \mathrm{MgCl}$ in $10 \mathrm{mM}$ Tris ( $\mathrm{pH}=8.0$ ). Sample was allowed to react for $30 \mathrm{~min}$ and then heated to $95{ }^{\circ} \mathrm{C}$ to stop reaction. Fluorescence conditions: $1 \mu \mathrm{M} 3^{\prime}, 3^{\prime}-\mathrm{cG}(\mathrm{d} 2 \mathrm{AP}) \mathrm{MP}, 15 \mathrm{mM} \mathrm{MnCl} 2,69 / 100$ dilution of the enzymatic reaction solution in $50 \mathrm{mM}$ Tris- $\mathrm{HCl}(\mathrm{pH}=7.5)$. Sample allowed to incubate with $3^{\prime}, 3^{\prime}-\mathrm{cG}(\mathrm{d} 2 \mathrm{AP}) \mathrm{MP}$ and $\mathrm{Mn}^{2+}$ for at least 30 min prior to fluorescence measurement.

case. Nonetheless the fluorescence of the probe, subjected to YybT cleavage, was $20 \%$ more than that of the uncleaved $3^{\prime} 3^{\prime} \mathrm{cG}(\mathrm{d} 2 \mathrm{AP}) \mathrm{MP}$ after $140 \mathrm{~min}$ so the probe can also be used to discover inhibitors of c-di-AMP-specific PDE's. We conclude that this new fluorescent dinucleotide probe, $3^{\prime}, 3^{\prime}-\mathrm{cG}(\mathrm{d} 2 \mathrm{AP}) \mathrm{MP}$, is appropriate for monitoring the inhibition of PDEs by inhibitors because in the presence of inhibitors, such as EDTA for SVPD, the fluorescence signal in the presence of inhibitor and absence are different (see Fig. 11). ${ }^{43,44}$

\section{Conclusion}

We have demonstrated that cyclic dinucleotide analog containing 2-aminoprurine is a general probe that can be used to monitor the enzymatic activities of PDE and DGC and could be a useful tool for high-throughput assays that seek to discover new inhibitors of c-di-GMP metabolism enzymes. ${ }^{45,46}$ Both $3^{\prime}, 3^{\prime}$-cGAMP and $2^{\prime}, 3^{\prime}$-cGAMP have been observed in nature and the distinction between the two is non-trivial and requires
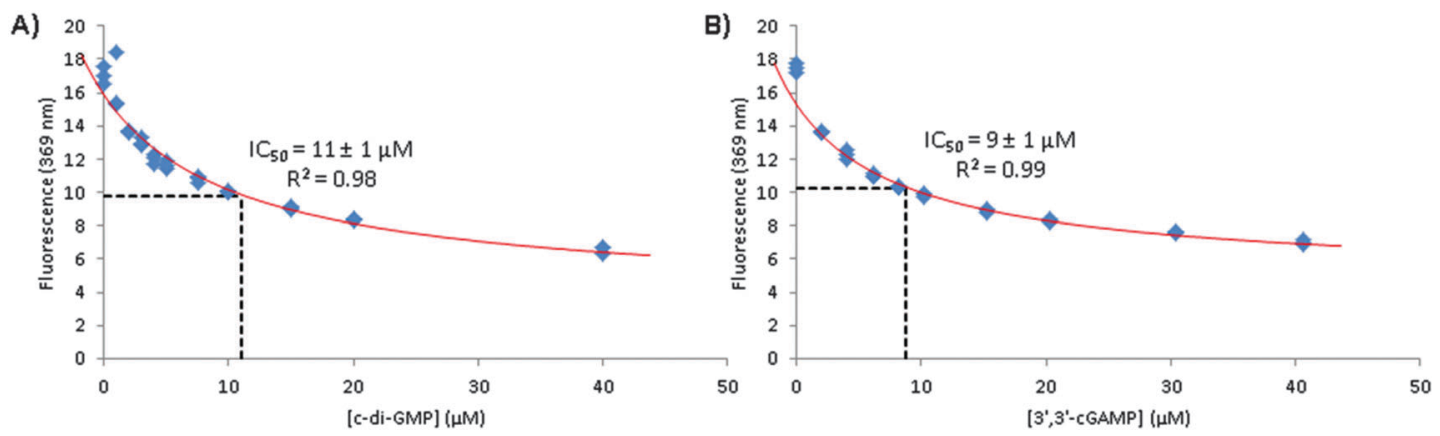

Fig. 9 A plot of fluorescence intensity (from $3^{\prime}, 3^{\prime}-c G(d 2 A P) M P$ ) versus concentration of c-di-NMP in the presence of $15 \mathrm{mM} \mathrm{MnCl} 2$ and fitted using eqn (S9) (ESI $\dagger$ ) to obtain IC 50 values of (A) c-di-GMP and (B) $3^{\prime}, 3^{\prime}$-cGAMP binding with $3^{\prime}, 3^{\prime}-\mathrm{cG}(\mathrm{d} 2 \mathrm{AP}) \mathrm{MP} .{ }^{42}$ Conditions: $1 \mu \mathrm{M} 3^{\prime}, 3^{\prime}-\mathrm{cG}(\mathrm{d} 2 \mathrm{AP}) \mathrm{MP}, 15 \mathrm{mM}$ $\mathrm{MnCl}_{2}, 50 \mathrm{mM}$ Tris- $\mathrm{HCl}(\mathrm{pH}=7.5)$ and varying concentration of cyclic dinucleotide. 


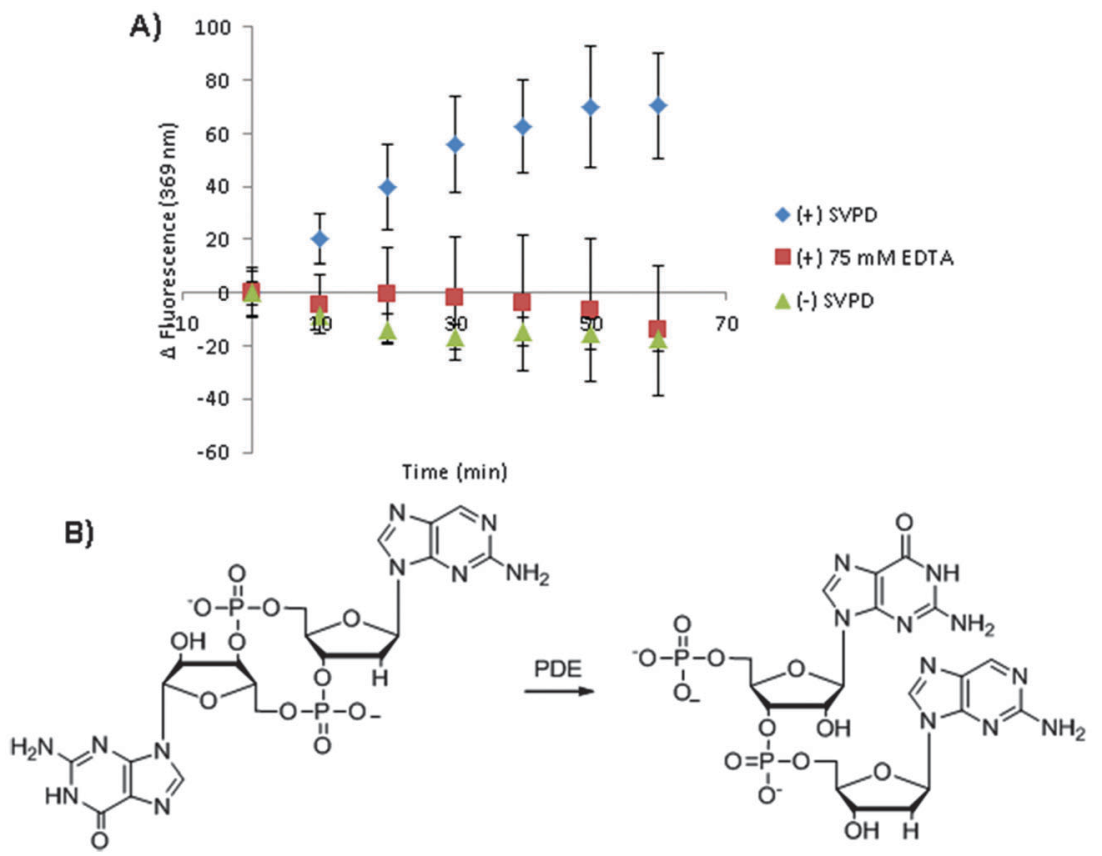

Fig. 11 (A) Cleavage of $3^{\prime}, 3^{\prime}-c G(d 2 A P) M P$ by the PDE, SVPD, indicated by the fluorescence emission of $3^{\prime}, 3^{\prime}-c G(d 2 A P) M P$ at $369 \mathrm{~nm}(\Delta \mathrm{Fluorescence}=$ $F-F_{0}$; where $F_{0}$ is initial fluorescence emission at time and $F$ is fluorescence emission at time $=t$ min). Blue diamonds: enzymatic reaction; red squares: enzymatic reaction plus the EDTA, an inhibitor; green triangles: reaction without enzyme. (B) Scheme depicting $3^{\prime}, 3^{\prime}-\mathrm{cG}(\mathrm{d} 2 \mathrm{AP}) \mathrm{MP}$ cleavage by a PDE. Conditions: (A) $1 \mu \mathrm{M}$ 3', 3'-cG(d2AP)MP, $15 \mathrm{mM} \mathrm{MgCl}, 0.001$ units per $\mathrm{mL}$ SVPD in $50 \mathrm{mM}$ Tris- $\mathrm{HCl}(\mathrm{pH}=8.5)$.

careful HPLC separation followed by MS-MS analysis. $3^{\prime}, 3^{\prime}-\mathrm{cG}(\mathrm{d} 2 \mathrm{AP}) \mathrm{MP}$ can discriminate between the $2^{\prime}, 3^{\prime}$ - and $3^{\prime}, 3^{\prime}$-forms of cGAMP and could be used as a confirmatory probe, whenever the phosphodiester linkage of an isolated cGAMP molecule comes into question.

Cyclic dinucleotides are known to aggregate into different polymorphs and it has been shown that both the sugar backbone and nature of nucleobase affect polymorphism. ${ }^{47,48}$ Recently, it has also been demonstrated that both c-di-GMP and c-di-AMP interact with diverse $\pi$-systems to form supramolecular aggregates. ${ }^{23-26,37,49}$ Herein, we use the fluorescent analog to show that under appropriate conditions, c-di-NMPs can also associate with each other to form heterodimers with $K_{\mathrm{d}}$ 's that are similar to cytoplasmic concentrations. Considering that some bacteria (such as $V$. Cholerae) are known to make both c-di-GMP and $3^{\prime}, 3^{\prime}$-cGAMP, ${ }^{3,10}$ could it be possible that beyond binding of these nucleotides to their respective receptors, different cyclic dinucleotides also directly associate (or "communicate") with each other and intracellular aromatic molecules to affect signaling? Or bacteria might possess the ability to spatial separate these signaling molecules in the cell, even though they do not contain membrane-bound organelles. Cyclic dinucleotide signaling might have another complex layer of interactions that might not be fully appreciated.

\section{Acknowledgements}

Funding for this work was provided by the National Science Foundation (CHE1307218), Camille Dreyfus Foundation,
GAANN fellowship (BTR), Millard and Lee Alexander Fellowship in Chemistry (BTR) and the University of Maryland's Dean's graduate fellowship (JZ).

\section{References}

1 S. L. Chua, S. Y. Tan, M. T. Rybtke, Y. Chen, S. A. Rice, S. Kjelleberg, T. Tolker-Nielsen, L. Yang and M. Givskov, Antimicrob. Agents Chemother., 2013, 57, 2066-2075.

2 R. Paul, S. Weiser, N. C. Amiot, C. Chan, T. Schirmer, B. Giese and U. Jenal, Genes Dev., 2004, 18, 715-727.

3 A. D. Tischler and A. Camilli, Mol. Microbiol., 2004, 53, 857-869.

4 R. W. McKee, M. R. Mangalea, E. B. Purcell, E. K. Borchardt and R. Tamayo, J. Bacteriol., 2013, 195, 5174-5185.

5 D. Kalia, G. Merey, S. Nakayama, Y. Zheng, J. Zhou, Y. Luo, M. Guo, B. T. Roembke and H. O. Sintim, Chem. Soc. Rev., 2013, 42, 305-341.

6 K. D. Smith, S. V. Lipchock, T. D. Ames, J. Wang, R. R. Breaker and S. A. Strobel, Nat. Struct. Mol. Biol., 2009, 16, 1218-1223.

7 J. W. Nelson, N. Sudarsan, K. Furukawa, Z. Weinberg, J. Wang and R. R. Breaker, Nat. Chem. Biol., 2013, 9, 834-839.

8 Y. Luo, J. Zhou, J. Wang, T. K. Dayie and H. O. Sintim, Mol. BioSyst., 2013, 9, 1535-1539.

9 K. D. Smith, C. A. Shanahan, E. L. Moore, A. C. Simon and S. A. Strobel, Proc. Natl. Acad. Sci. U. S. A., 2011, 108, 7757-7762.

10 B. W. Davies, R. W. Bogard, T. S. Young and J. J. Mekalanos, Cell, 2012, 149, 358-370. 
11 Q. Yin, Y. Tian, V. Kabaleeswaran, X. Jiang, D. Tu, M. J. Eck, Z. J. Chen and H. Wu, Mol. Cell, 2012, 46, 735-745.

12 T. S. Xiao and K. A. Fitzgerald, Mol. Cell, 2013, 51, 135-139.

13 P. Gao, M. Ascano, Y. Wu, W. Barchet, B. L. Gaffney, T. Zillinger, A. A. Serganov, Y. Liu, R. A. Jones, G. Hartmann, T. Tuschl and D. J. Patel, Cell, 2013, 153, 1094-1107.

14 X. Zhang, H. Shi, J. Wu, X. Zhang, L. Sun, C. Chen and Z. J. Chen, Mol. Cell, 2013, 51, 226-235.

15 L. Sun, J. Wu, F. Du, X. Chen and Z. J. Chen, Science, 2013, 339, 786-791.

16 D. Gao, J. Wu, Y.-T. Wu, F. Du, C. Aroh, N. Yan, L. Sun and Z. J. Chen, Science, 2013, 341, 903-906.

17 I. Kiburu, A. Shurer, L. Yan and H. O. Sintim, Mol. Biosyst., 2008, 4, 518-520.

18 H. C.-Y. Jenny, D. Don and J. Oger, Nucleosides Nucleotides, 1985, 4, 377-389.

19 O. Magdenoska, J. Martinussen, J. Thykaer and K. F. Nielsen, Anal. Biochem., 2013, 440, 166-177.

20 V. Stelitano, A. Brandt, S. Fernicola, S. Franceschini, G. Giardina, A. Pica, S. Rinaldo, F. Sica and F. Cutruzzola, Nucleic Acids Res., 2013, 41, e79.

21 S. Nakayama, Y. L. Luo, J. Zhou, T. K. Dayie and H. O. Sintim, Chem. Commun., 2012, 48, 9059-9061.

22 H. Gu, K. Furukawa and R. R. Breaker, Anal. Chem., 2012, 84, 4935-4941.

23 S. Nakayama, I. Kelsey, J. Wang, K. Roelofs, B. Stefane, Y. Luo, V. T. Lee and H. O. Sintim, J. Am. Chem. Soc., 2011, 133, 4856-4864.

24 S. Nakayama, K. Roelofs, V. T. Lee and H. O. Sintim, Mol. Biosyst., 2012, 8, 726-729.

25 S. Nakayama, I. Kelsey, J. Wang and H. O. Sintim, Chem. Commun., 2011, 47, 4766-4768.

26 B. T. Roembke, J. X. Wang, S. Nakayama, J. Zhou and H. O. Sintim, RSC Adv., 2013, 3, 6305-6310.

27 M. T. Rybtke, B. R. Borlee, K. Murakami, Y. Irie, M. Hentzer, T. E. Nielsen, M. Givskov, M. R. Parsek and T. TolkerNielsen, Appl. Environ. Microbiol., 2012, 78, 5060-5069.

28 C. A. Kellenberger, S. C. Wilson, J. Sales-Lee and M. C. Hammond, J. Am. Chem. Soc., 2013, 135, 4906-4909.

29 D. Antoniani, P. Bocci, A. Maciag, N. Raffaelli and P. Landini, Appl. Microbiol. Biotechnol., 2010, 85, 1095-1104.

30 M. Egli, R. V. Gessner, L. D. Williams, G. J. Quigley, G. A. van der Marel, J. H. van Boom, A. Rich and C. A. Frederick, Proc. Natl. Acad. Sci. U. S. A., 1990, 87, 3235-3239.
31 See PDB codes: $3 \mathrm{I} 5 \mathrm{~A}$ and $4 \mathrm{ETZ}$.

32 S. O. Kelley and J. K. Barton, Science, 1999, 283, 375-381.

33 O. J. G. Somsen, V. A. Hoek and V. H. Amerongen, Chem. Phys. Lett., 2005, 402, 61-65.

34 S. Bharill, P. Sarkar, J. D. Ballin, I. Gryczynski, G. M. Wilson and Z. Gryczynski, Anal. Biochem., 2008, 377, 141-149.

35 J. M. Jean and K. B. Hall, Proc. Natl. Acad. Sci. U. S. A., 2001, 98, 37-41.

36 M. Gentner, M. G. Allan, F. Zaehringer, T. Schirmer and S. Grzesiek, J. Am. Chem. Soc., 2012, 134, 1019-1029.

37 I. Kelsey, S. Nakayama and H. O. Sintim, Bioorg. Med. Chem. Lett., 2012, 22, 881-885.

38 M. J. J. Blommers, C. A. G. Haasnoot, J. Walters, G. A. van der Marel, J. H. van Boom and C. W. Hilbers, Biochemistry, 1988, 27, 8361-8369.

39 B. Christen, M. Christen, R. Paul, F. Schmid, M. Folcher, P. Jenoe, M. Meuwly and U. Jenal, J. Biol. Chem., 2006, 281, 32015-32024.

40 G. Witte, S. Hartung, K. Buttner and K. P. Hopfner, Mol. Cell, 2008, 30, 167-178.

41 P. Ross, H. Weinhouse, Y. Aloni, D. Michaeli, P. WeinbergerOhana, R. Mayer, S. Braun, E. de Vroom, G. A. van der Marel, J. H. van Boom and M. Benziman, Nature, 1987, 325, 279-281.

42 Y. Wang, K. Hamasaki and R. R. Rando, Biochemistry, 1997, 36, 768-779.

43 S. S. Al-Saleh and S. Khan, Prep. Biochem. Biotechnol., 2011, 41, 262-277.

44 D. Amikam and M. Benziman, J. Bacteriol., 1989, 171, 6649-6655.

45 K. Sambanthamoorthy, R. E. Sloup, V. Parashar, J. M. Smith, E. E. Kim, M. F. Semmelhack, M. B. Neiditch and C. M. Waters, Antimicrob. Agents Chemother., 2012, 56, 5202-5211.

46 K. Sambanthamoorthy, C. Luo, N. Pattabiraman, X. Feng, B. Koestler, B. Koestler, C. M. Waters and T. J. Palys, Biofouling, 2014, 30, 17-28.

47 J. Wang, J. Zhou, G. P. Donaldson, S. Nakayama, L. Yan, Y.-F. Lam, V. T. Lee and H. O. Sintim, J. Am. Chem. Soc., 2011, 133, 9320-9330.

48 J. Zhou, D. A. Sayre, J. X. Wang, N. Pahadi and H. O. Sintim, Molecules, 2012, 17, 13376-13389.

49 J. Zhou, D. A. Sayre, Y. Zheng, H. Szmacinski and H. O. Sintim, Anal. Chem., 2014, 86, 2412-2420. 